\title{
Analysis of TMT primary mirror control-structure interaction
}

Douglas G. MacMynowski, Peter M. Thompson, Mark J. Sirota

Douglas G. MacMynowski, Peter M. Thompson, Mark J. Sirota, "Analysis of TMT primary mirror control-structure interaction," Proc. SPIE 7017, Modeling, Systems Engineering, and Project Management for Astronomy III, 701715 (9 July 2008); doi: 10.1117/12.787003

Event: SPIE Astronomical Telescopes + Instrumentation, 2008, Marseille, France 


\title{
Analysis of TMT Primary Mirror Control-Structure Interaction
}

\author{
Douglas G. MacMynowski ${ }^{a}$, Peter M. Thompson ${ }^{b}$ and Mark J. Sirota ${ }^{c}$ \\ ${ }^{a}$ California Institute of Technology \\ Department of Control and Dynamical Systems, Pasadena CA 91125 \\ ${ }^{b}$ Systems Technology, Inc., Hawthorne, CA 90250 \\ ${ }^{c}$ Thirty Meter Telescope Observatory, Pasadena, CA 91125
}

\begin{abstract}
The primary mirror control system (M1CS) keeps the 492 segments of the Thirty Meter Telescope primary mirror aligned in the presence of disturbances. A global position control loop uses feedback from inter-segment edge sensors to three actuators behind each segment that control segment piston, tip and tilt. If soft force actuators are used (e.g. voice-coil), then in addition to the global position loop there will be a local servo loop to provide stiffness. While the M1 control system at Keck compensates only for slow disturbances such as gravity and thermal variations, the M1CS for TMT will need to provide some compensation for higher frequency wind disturbances in order to meet stringent error budget targets. An analysis of expected high-wavenumber wind forces on M1 suggests that a $1 \mathrm{~Hz}$ control bandwidth is required for the global feedback of segment edge-sensorbased position information in order to minimize high spatial frequency segment response for both seeing-limited and adaptive optics performance. A much higher bandwidth is required from the local servo loop to provide adequate stiffness to wind or acoustic disturbances. A related paper presents the control designs for the local actuator servo loops. The disturbance rejection requirements would not be difficult to achieve for a single segment, but the structural coupling between segments mounted on a flexible mirror cell results in controlstructure interaction (CSI) that limits the achievable bandwidth. Using a combination of simplified modeling to build intuition and the full telescope finite element model for verification, we present designs and analysis for both the local servo loop and global loop demonstrating sufficient bandwidth and resulting wind-disturbance rejection despite the presence of CSI.
\end{abstract}

\section{Keywords: Extremely Large Telescopes, Control Systems, Control-Structure-Interaction}

\section{INTRODUCTION}

The primary mirror (M1) of the Thirty Meter Telescope (TMT) is composed of 492 hexagonal segments of circumscribed diameter $1.43 \mathrm{~m}$, as shown in Figure 1. The out-of-plane degrees of freedom are controlled by the primary mirror control system (M1CS). The overall approach is similar to that used to control the positions of the 36 segments in each of the Keck telescopes primary mirrors. ${ }^{1,2}$ However, while the Keck control system compensates only for low frequency disturbances such as thermal variations or the changing orientation of the mirror with respect to gravity, the TMT M1CS will also compensate for some wind-induced motion of the M1 segments. This results in a significantly higher bandwidth requirement, which in turn leads to the potential for control-structure-interaction (CSI): ${ }^{3,4}$ undesirable dynamic interaction between the primary mirror control system and the telescope structural dynamics.

The M1CS bandwidth requirements are driven by estimates of the disturbance environment, in particular the unsteady wind forces, and possibly acoustic forces at higher frequencies. In order to minimize seeing within the dome and above M1 due to thermal variations, the TMT enclosure will be vented, with the vent opening chosen to maintain a mean wind speed of approximately $1 \mathrm{~m} / \mathrm{s}$ across M1 when possible. This results in $\sim 0.5 \mathrm{~Pa}$ dynamic pressure on M1 (at 3000m ASL), with most of the wind energy below $1 \mathrm{~Hz}$. Setting a $10 \mathrm{~N} / \mu \mathrm{m}$ stiffness requirement below $1 \mathrm{~Hz}$ on the entire M1 support system results in segment displacements of order $65 \mathrm{~nm}$ due to wind; at low spatial frequencies there will be even higher displacements due to the compliance of the telescope structure. While much of this response is highly correlated between neighbouring segments, an M1CS control bandwidth of $1 \mathrm{~Hz}$ is required ${ }^{5}$ to reduce the wind-induced mirror response both for seeing-limited performance and to meet the desired error budget allocation of $10 \mathrm{~nm}$ rms wavefront error uncorrectable by adaptive optics.

Modeling, Systems Engineering, and Project Management for Astronomy III, edited by George Z. Angeli, Martin J. Cullum, Proc. of SPIE Vol. 7017, 701715, (2008) · 0277-786X/08/\$18 · doi: 10.1117/12.787003 

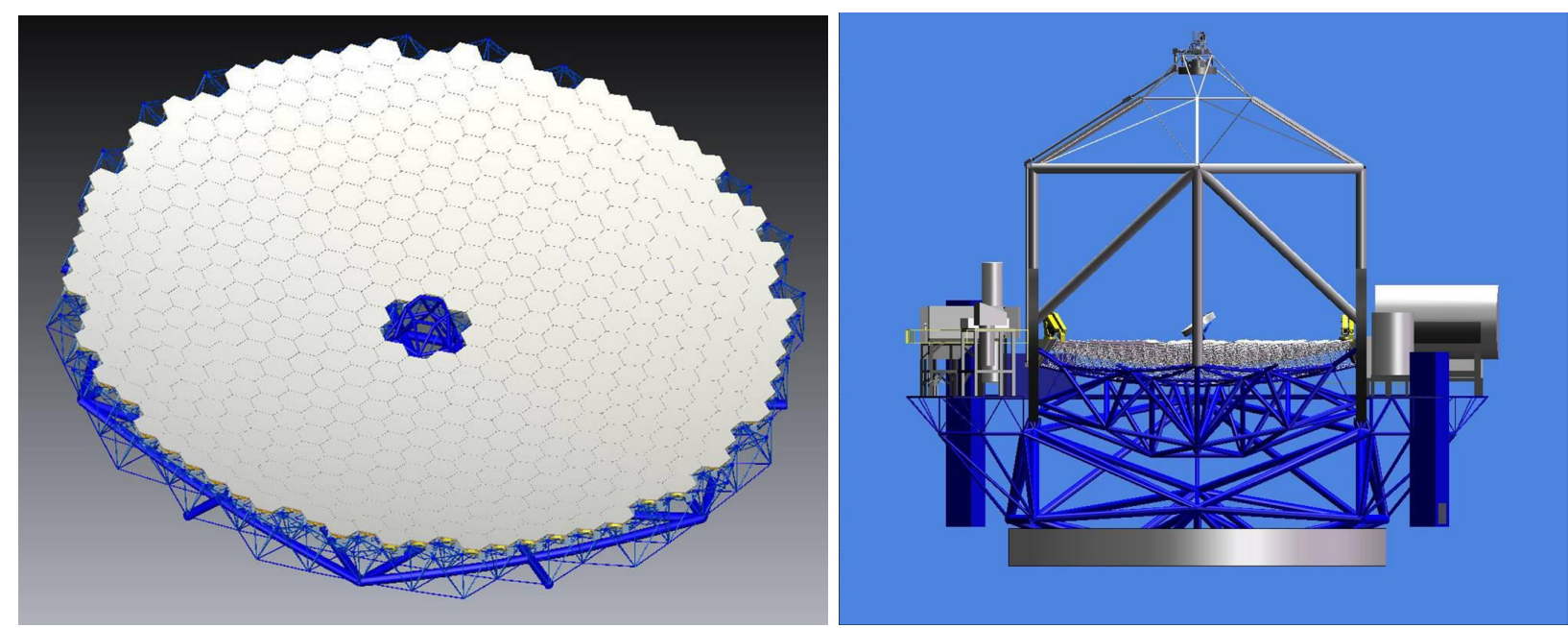

Figure 1. Left: TMT primary mirror, composed of 492 hexagonal segments mounted on a flexible mirror cell. Right: TMT telescope structure.

The Keck M1CS uses "hard" actuators that provide significant stiffness without power. However, a "soft" actuator based on a voice-coil has significant potential advantages in cost, reliability, and the ability to add damping to segment support modes. A local servo control loop using feedback of the actuator output displacement can be used to provide the required stiffness at low frequency; this control system is discussed in more detail in a companion paper. ${ }^{6}$ Both actuator approaches are compared herein. With either actuator approach, a global control loop is used to maintain the position of the mirror segments, using feedback from edge sensors that measure the relative motion between neighbouring segments. Herein we assume that we can estimate the segment positions from these edge sensor measurements, ${ }^{7}$ with only the overall mirror piston, tip and tilt being unobservable. The effect of this estimation on sensor noise is well understood; the effect of errors in the estimation on the dynamics will be the subject of future work.

While both the local (actuator servo) and global position control loops would be straightforward to design if the segments were supported by a rigid backplane, the flexibility of the mirror cell and telescope structure lead to coupling between the segments that can potentially lead to instability. The next section introduces this CSI issue in more detail, and in particular describes the approach used herein to reduce the computational complexity to manageable levels. The key insight is that a diagonal system of identical subsystems is diagonal under any change in basis, and thus the dynamics of the 492 segments and their control can be projected onto a Zernike basis. Section 3 gives the relevant background on both the segment support and telescope structural dynamics. Design of the control loops is presented in section 4. While Ref. [6] provides most of the details on the local servo control system, sufficient background is summarized here. The interactions between these control loops and the telescope structural dynamics is then presented in section 5. The analysis approach and conclusions should also be relevant to other segmented large telescope designs.

\section{CONTROL STRUCTURE INTERACTION}

It is clear that a control system that is designed assuming that a segment is mounted on a rigid support can lead to problems if the segment is actually mounted on a flexible structure. It is less clear how this problem scales with the number and mass of segments. Some studies suggest that the problem scales roughly linearly with the number of control loops. ${ }^{4}$ Since CSI analysis for Keck ${ }^{1,8}$ suggested a $0.5 \mathrm{~Hz}$ maximum bandwidth with only 36 segments, this would be cause for concern for the 492 segment primary mirror of TMT. However, the linear scaling is true only for a given structure, and it is the ratio of the total mass of controlled segments to the mass of the mirror cell that is a more relevant parameter, ${ }^{6,9}$ because it is the total mass of controlled segments that determines the net force introduced into the coupling structure. That is, increasing the number of controlled segments does not affect stability if the areal density remains constant. Furthermore, in extrapolating from Keck results to TMT, note as discussed in section $4 \mathrm{~b}$ below, that the Keck analysis only considered pure integral control, and significantly higher bandwidth could have been achieved with additional effort. 

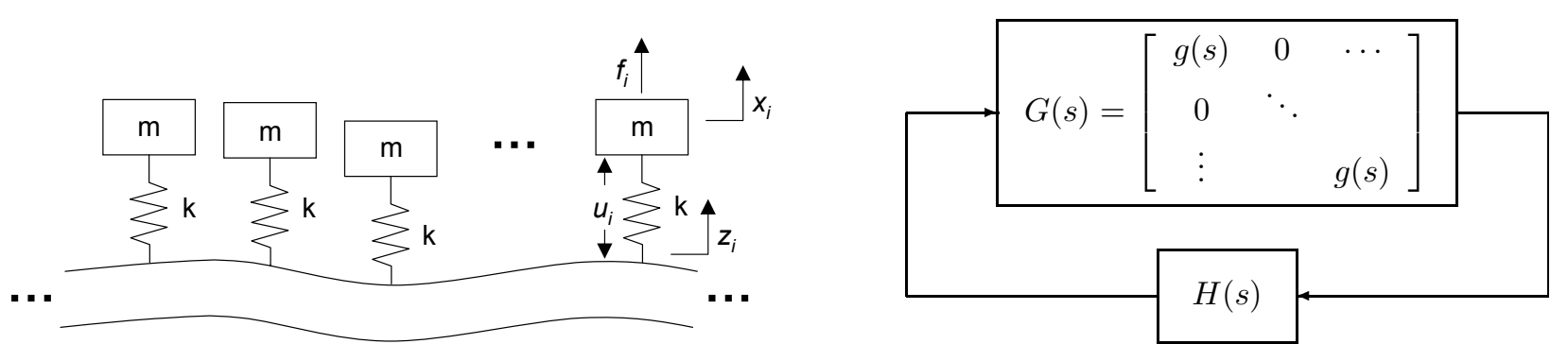

Figure 2. Schematic of $n$ identical oscillators coupled through a supporting structure (left), and block diagram representation (right): $H(s)$ captures the support dynamics, and the dynamics of each oscillator is given by $g(s)$.

The telescope control problem can be illustrated schematically as shown in Fig. 2; there are many identical subsystems coupled to each other through the telescope structure. The dynamics of the state space representation of such a system are described by $\dot{x}=A x$ with the $A$ matrix given in Eq. 1 .

$$
A=\left[\begin{array}{cccc}
A_{h} & \phi_{1}^{T} & \cdots & \phi_{n}^{T} \\
\phi_{1} & a & \cdots & 0 \\
\vdots & 0 & \ddots & 0 \\
\phi_{n} & 0 & 0 & a
\end{array}\right]
$$

where $a$ and $A_{h}$ describe the dynamics of an individual segment $(g(s)$ in Fig. 2) and the support structure $(H(s)$ in Fig. 2) respectively. With the dynamics of all 492 segments included, the resulting matrix for TMT will be quite large, and the eigenvalues cannot be easily solved for. Instead, we look for an approach to simplifying the computation while providing useful intuition.

First, consider the case where the coupling to the structure is the same for every segment (or differs only by a scalar factor). Then it is straightforward to show that the $A$ matrix in Eq. (1) can be transformed through a unitary transformation $V^{T} A V$ to yield $n-1$ copies of the uncoupled segment dynamics, and a block $A_{V}$ that captures the coupled system dynamics. The matrix $V$ is given by

$$
V=\left[\begin{array}{cccc}
I & 0 & \cdots & 0 \\
0 & \vdots & \vdots & \\
\vdots & \frac{b}{\sqrt{n}} \otimes I & \mathcal{C} \otimes I & \cdots \\
0 & \vdots & \vdots &
\end{array}\right] \quad \text { and } \quad A_{V}=\left[\begin{array}{cc}
A_{h} & \sqrt{n} \phi^{T} \\
\sqrt{n} \phi & a
\end{array}\right]
$$

with $I$ and 0 being the identity and zero matrix respectively of appropriate dimension, $b \in \mathbb{R}^{n}$ the vector of ones, $\mathcal{C} \in \mathbb{R}^{n \times(n-1)}$ an orthogonal complement to $b$, and $\otimes$ the Kronecker product.

The vector $b$ in $V$ describes the mode shape of the coupling. The generalization of the above result is to note that a diagonal system of identical subsystems remains diagonal under any change of basis. Thus for any unitary matrix $\phi$ (so $\left.\phi^{T} \phi=I\right)$ then $\phi^{T} G \phi=G$. If the structural dynamics were described solely by the displacements at the segment locations, then the modes of the structure evaluated at these locations would provide an orthogonal basis for transforming the segment dynamics. The transformation would result in $n$ decoupled systems that each describes the coupling between the segment dynamics and one of the structural modes, as with $A_{V}$ in Eq. (2) above. However, in general, the coupling structural dynamics involve additional degrees of freedom, and thus the structural mode shapes will not provide an orthonormal basis when evaluated at the segment locations.

Instead of using a basis derived from the structural dynamics, we will instead use the Zernike basis set. This has two drawbacks relative to using the structural modes. First, any given structural mode will in general have non-zero projection onto many basis vectors, and conversely, any basis vector will include dynamics associated with many modes. Second, there is no reason to expect the dynamics associated with one basis vector to be decoupled from those of another, and so the different components cannot be analyzed in isolation as was true in the simple case illustrated in Eq. (2). If we included the first 492 Zernike basis vectors, there would be no 
computational savings relative to the original untransformed system. However, there is still an advantage in using the Zernike basis because the stability characteristics can be accurately predicted with relatively few basis vectors included. This results from the fact that the coupling, and therefore the control-structure-interaction, is dominated by the most compliant, lowest frequency modes of the supporting structure, and these are the longest wavelength, lowest wavenumber modes, and predominantly project onto the lowest order Zernike basis vectors. For high wavenumber motion involving significant relative motion between neighbouring segments, the support structure is relatively stiff.

\section{STRUCTURAL DYNAMICS}

The TMT telescope structure is shown in Fig. 1 and the finite element modeling described in [10]. All analyses herein will be presented for a $30^{\circ}$ zenith angle. Unless otherwise noted, the damping of the telescope structure is assumed to be $0.5 \%$, based on estimates from VLT and Gemini. In order to maintain adequate detail on the dynamics of each segment support, the segment dynamics are not included in the overall telescope model, but will be added in separately as needed. Thus, when we refer to the "telescope structure", this includes masses for the mirror segments (which must be subtracted out to avoid doublebookkeeping) but these are connected to the mirror cell with infinite-stiffness links.

The mirror cell itself consists of three layers of truss structure of increasing spatial coarseness. The top layer provides some local compliance so that a force

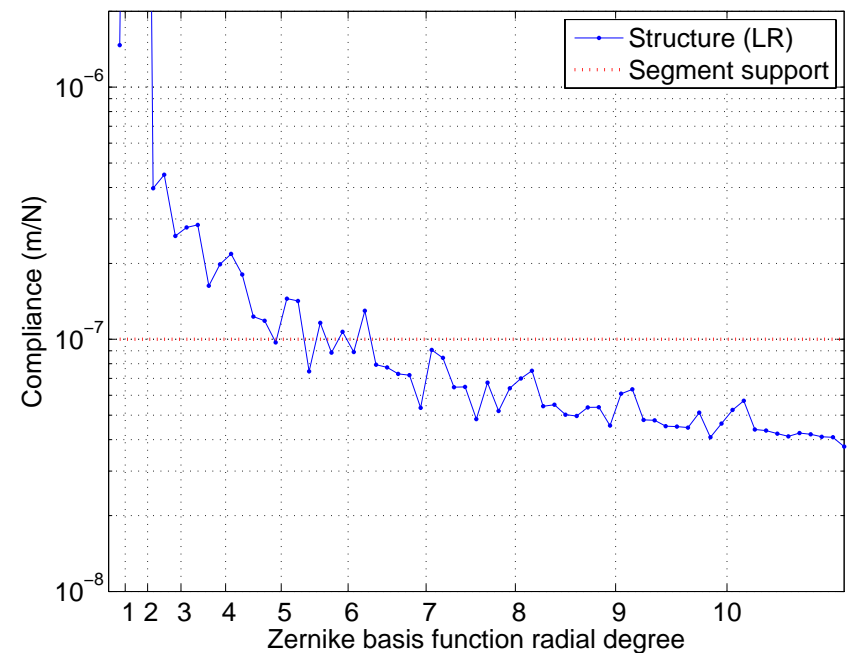

Figure 3. Compliance of telescope structure on Zernike basis, compared with segment support compliance.

on one segment results in some displacement only of that particular segment. The coarser truss layers of the mirror cell distribute loads, so that a force on one segment predominantly results in a spatially smooth displacement pattern. The tip and tilt dynamics of the telescope are significantly altered due to the presence of the mount control system ${ }^{11}$ (included in the following analyses) and at very low frequencies (below $\sim 0.2 \mathrm{~Hz}$ ) will also depend on the optical guide loops (not included in the following analyses).

Of particular relevance to the M1CS CSI is the telescope structural dynamics projected onto a Zernike basis of force distributions on the primary mirror. The quasi-static compliance of the structure is shown in Fig. 3. This represents the normalized amplitude (spatial rms) due to a unit (spatial rms) load applied with a given Zernike basis force distribution. There is little cross-compliance between Zernike degrees of freedom. The primary observation from this figure is that, as expected, the structural compliance is largest at low wavenumber, and thus the coupling with the segment dynamics and control system will be largest at low wavenumber. At sufficiently high wavenumber, the response is dominated by the purely local compliance of the top layer of the mirror cell, which does not introduce any coupling between segments.

The dynamics of the structure, projected onto a Zernike basis, are shown in Fig. 4 . Note that the $p=1$ tip/tilt dynamics at low frequency interact with the mount control dynamics, resulting in the damped peak evident in the corresponding plot. The lowest frequency modes of the mirror cell are sufficiently similar to the Zernike basis vectors so that the dynamics associated with each Zernike basis vector involve relatively few dominant structural modes with minimum frequency typically increasing with radial degree.

In addition to the telescope structural dynamics, the control system depends on the segment support dynamics. With a rigid actuator, the dominant modes of this support are at $35 \mathrm{~Hz}$ and higher (with the exception of a clocking mode that does not influence either control design or optical response). With a soft actuator, the modes that limit the control bandwidth are above $85 \mathrm{~Hz}$.

\subsection{Local servo loop}

\section{CONTROL DESIGN}

Details of the requirements, dynamics, and design issues for the local servo control loop for the soft (voice-coil) actuator are presented in [6] and will not be duplicated here. However, because the local servo loop has the 

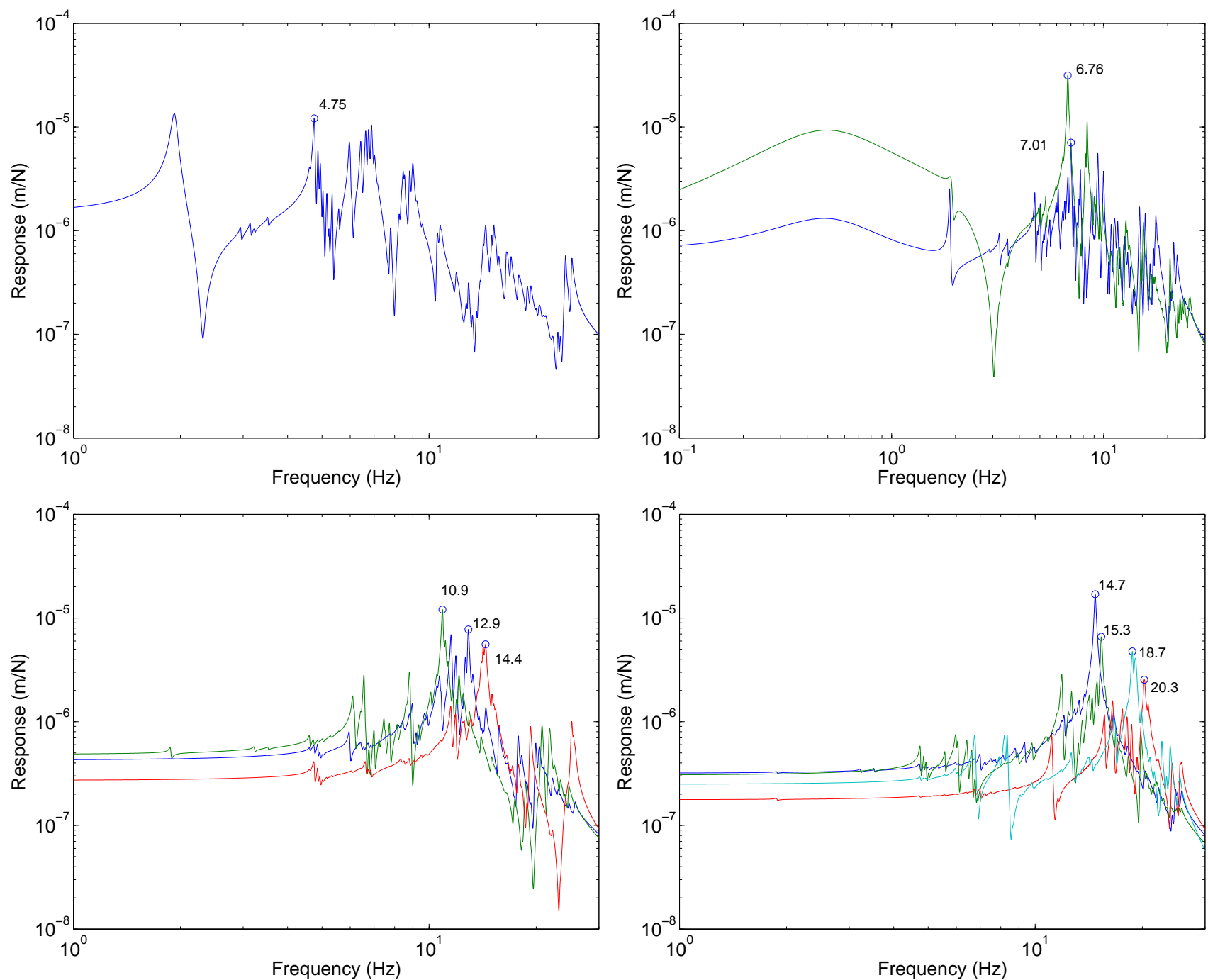

Figure 4. Transfer function of structure to Zernike inputs of radial degree $p=0$ (piston, top left), $p=1$ (tip/tilt, top right), $p=2$ and $p=3$ (bottom row). Key modal frequencies are identified; the relevant frequencies increase with Zernike radial degree.

potential to interact with the structural dynamics, a brief summary is relevant. In particular, note that the servo loops can also be analyzed in a primary mirror Zernike basis. Figure 5 presents the open-loop transfer function from actuator force to actuator displacement for an actuator on a segment mounted on a rigid base, and for the projection onto the first few Zernike modes. Any control design must be stable for all of these cases. Note that the high-frequency dynamics in Fig. 5 are identical for all cases only because the modal solution used for the telescope model does not include modes above $35 \mathrm{~Hz}$. However, because the high-frequency compliance is probably dominated by that of the segment support assembly, the response will likely be similar except near transfer function zeros where the segment support appears dynamically stiff.

An additional observation regarding the local servo loops is that, with 1476 actuators, it is impractical to tune control loops for every segment, and the control will need to be designed from models before mounting all of the actuators into the telescope. This means that the control design must be extremely robust to uncertainties in the system model. Because the servo sensor and actuator are collocated (in the ideal case), the transfer function between the actuator command and the time-derivative of the sensor output will be positive real regardless of the structural dynamics and coupling, and a positive-real servo control design would guarantee stability regardless of knowledge of the coupling. While this is attractive, this would preclude the use of integral gain that is essential for maintaining low frequency stiffness. 

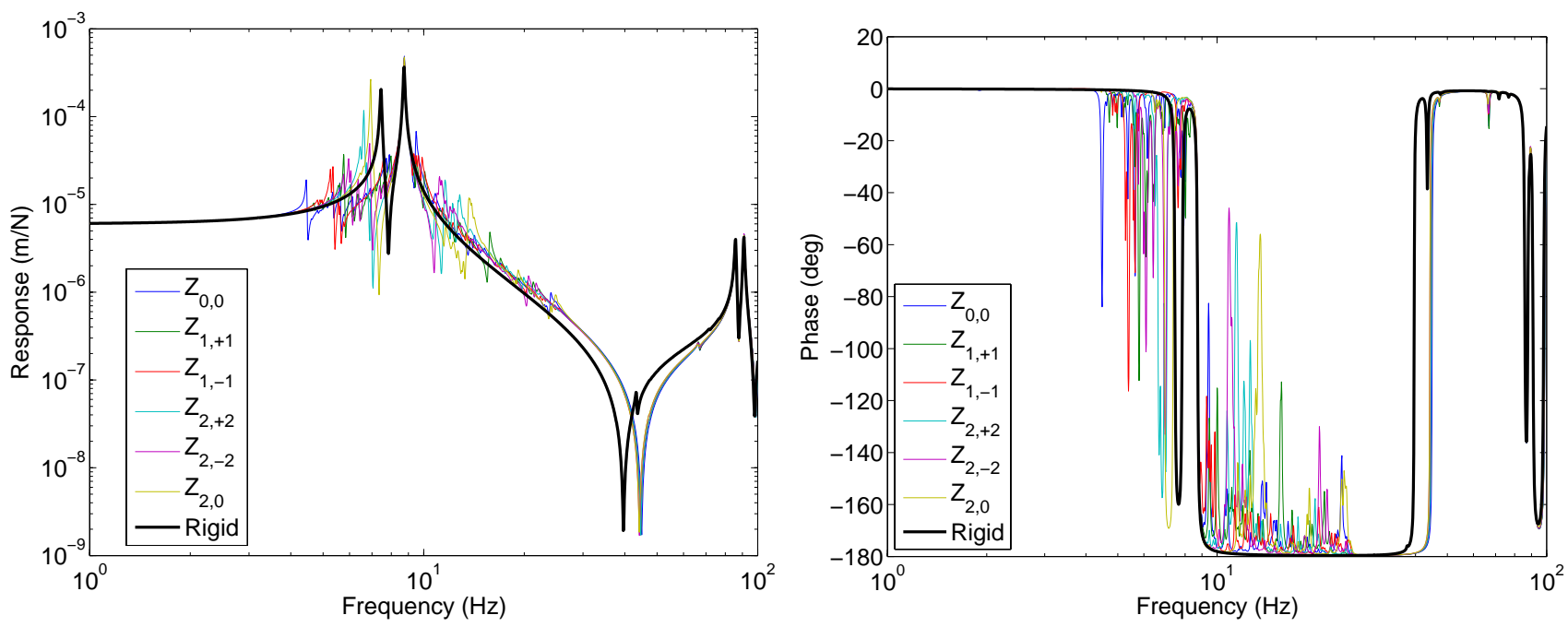

Figure 5. Actuator open-loop transfer function for segments mounted on the telescope structure, analyzed in a Zernike basis, and compared with the transfer function for a segment mounted on a rigid base. Magnitude (left) and phase (right).

Also note that it is not sufficient in designing the actuator servo loop to ensure stability for each of the Zernike-basis transfer functions in Fig. 5, because the coupling between Zernike bases is non-zero. Nonetheless, the Zernike basis provides both intuition on how to design the controller, and relatively few basis elements are required to predict stability both for the servo loop and for the global loop in the next subsection.

There are two strategies considered for the servo control design, depending on whether additional passive damping is added in parallel with the voice-coil actuator. The controller documented in $\lceil 6\rceil$ that assumes no damping has been added results in insufficient robustness to CSI when mounted on the actual mirror cell. Requiring that damping be added to the actuator opens up the design space and allows for a more robust servo design. Only the robustness of this latter approach is presented herein, as this is the more robust of the two strategies. The controller is a simple PI with additional lead compensation, shown in Fig. 6 with loop cross-over frequency of $24 \mathrm{~Hz}$ and $-3 \mathrm{~dB}$ bandwidth on command following of $17 \mathrm{~Hz}$. The control gain in Fig. 6 is the dynamic stiffness of the actuator, which does not quite meet the desired low frequency stiffness target of $10 \mathrm{~N} / \mu \mathrm{m}$.

Results that follow compare the soft actuator with servo loop to an idealized hard actuator; in this case, no servo control loop is designed, and it is assumed that the actuator responds perfectly to displacement commands.

\subsection{Global control}

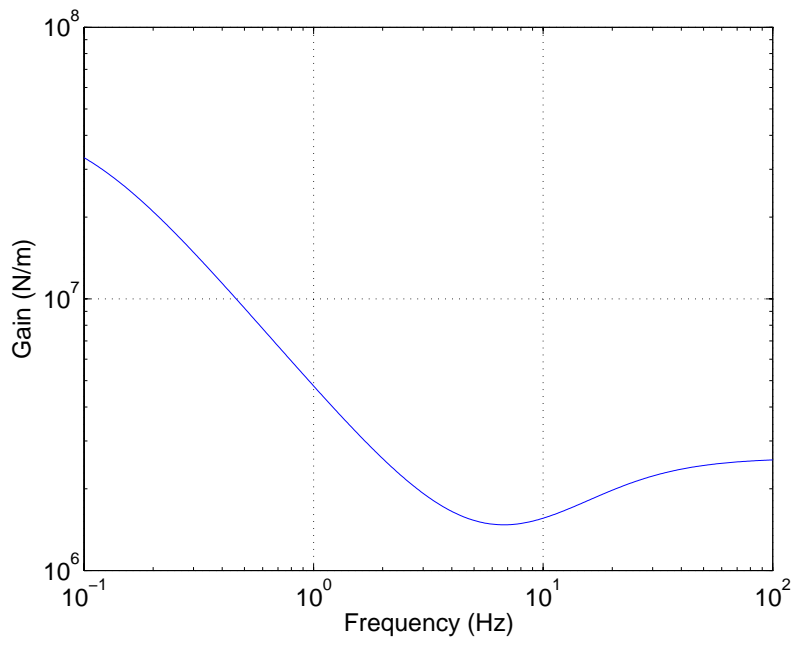

Figure 6. Control gain of actuator servo loop.

The global primary mirror control loop uses edge sensors to estimate the position of each mirror segment at the actuator locations, and collocated SISO control loops to minimize the mirror motion. ${ }^{7}$ As noted earlier, the transformation from segment displacements $x$ to edge sensor response $y=A x$ and back to segment displacements $\hat{x}=B y$ is assumed herein to be perfect, with the only effects being to introduce sensor noise (which depends on the spatial shape of the response, but does not affect dynamics or stability) and to project out the unobservable global piston, tip and tilt of the primary mirror. Errors in knowledge of $A$ can have a significant effect on the estimation of low spatial-frequency primary mirror responses, but this is not considered herein.

The global control can be analyzed in a Zernike basis, and if identical controllers are used at each location to minimize the estimated displacement $\hat{x}$, then the control bandwidths will also be identical in Zernike space. However, it is straightforward to use a "modal" control approach by transforming $\hat{x}$ into Zernike space, designing 

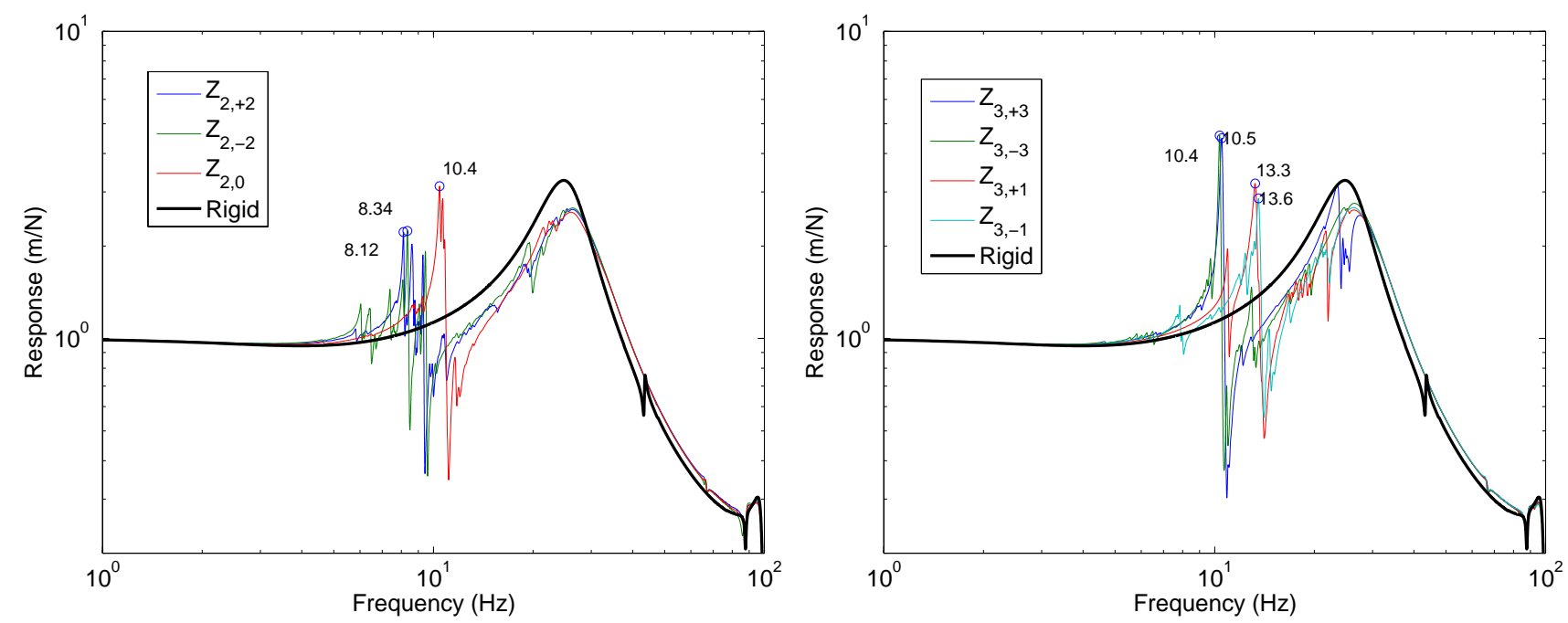

Figure 7. Global control open-loop transfer function, for Zernike radial degree $p=2$ (left) and $p=3$ (right), compared with transfer function for rigid base (solid black line).
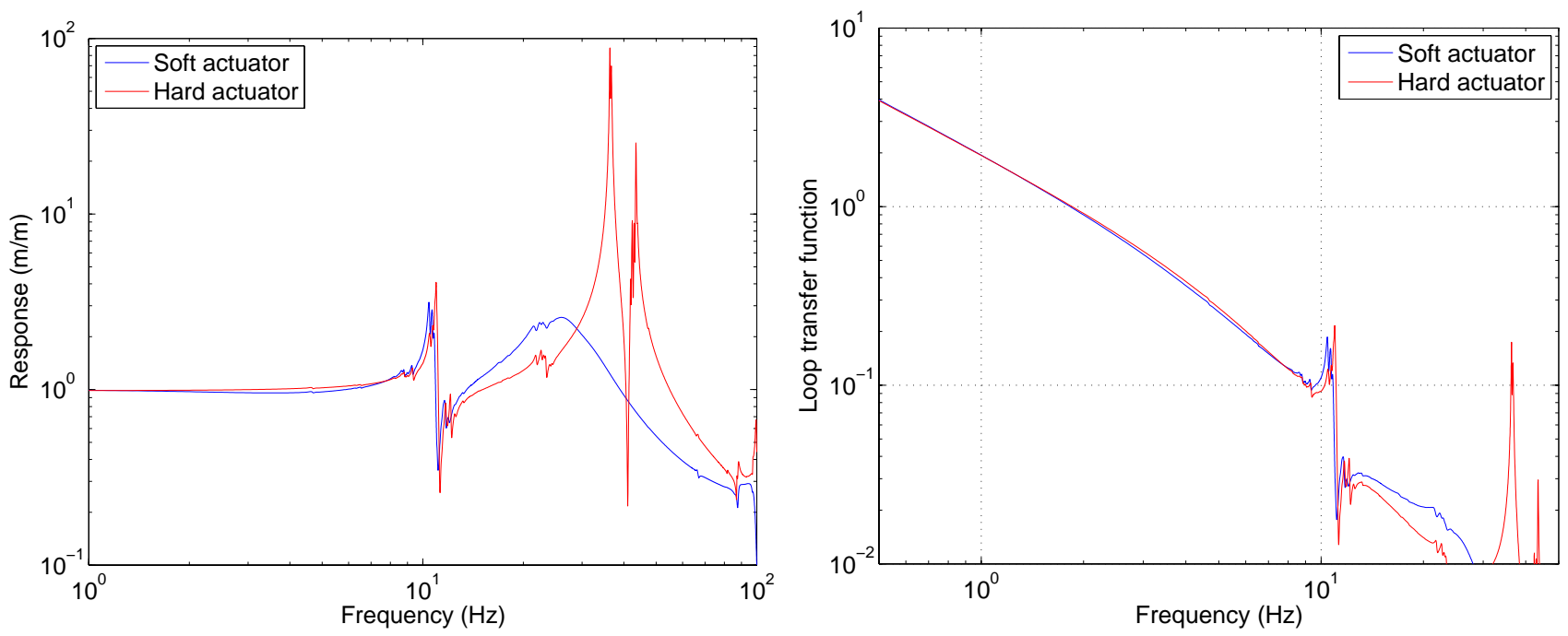

Figure 8. Global control open-loop (left) and loop (right) transfer function for focus-mode (Zernike 2,0) comparing the soft actuator stiffened with a servo loop to an ideal hard actuator. The loop transfer function assumes a $1.35 \mathrm{~Hz}(-3 \mathrm{~dB}$ sensitivity) bandwidth with a 2-pole $7 \mathrm{~Hz}$ rolloff.

control that differs for different Zernike modes, and transforming the resulting control command back into actuator space. The singular value decomposition of the geometric transformation matrix $A=U S V^{T}$ also provides a basis for representing or designing the control; the first few hundred basis vectors closely resemble Zernike basis vectors. ${ }^{13}$ Using this basis rather than a Zernike basis would allow the control gain (but not other dynamics of the control law) to be varied for different modes simply by altering the $B$ matrix. Since the results of section 5 below indicate that it is only the first few global modes that would require a reduced gain, either basis set is equivalent for this purpose.

Figure 7 gives the open-loop transfer function from actuator displacement command to mirror position, comparing the ideal response for a single segment mounted on a rigid base to the response aligned with the first few Zernike basis vectors that are observable with the edge sensor (radial degree 2 and higher).

Figure 8 compares the focus-mode response for the soft and hard actuator, and also gives the loop transfer function in order to illustrate the relative importance of different peaks in the response. The soft actuator provides damping to the segment support resonances, but otherwise, the transfer functions are similar and thus the achievable global control bandwidths in the presence of CSI should be expected to be similar. 
Because only a low bandwidth was required at Keck, the global M1CS loop at Keck uses pure integral control. Since the bandwidth is limited by the gain-margin from lightly damped structural modes at frequencies well above the control bandwidth, significantly higher bandwidths can be achieved by adding roll-off to the compensator. An alternate strategy would be to include a non-zero position gain instead of a roll-off, and rely on phase-stability rather than gain-stability for all of the structural mode interactions. This would then require that phase-stability be maintained to a sufficiently high frequency, and thus would require a high sampling rate and minimal lags in electronics and filtering.

A Butterworth structural filter is used to maximize the high-frequency gain reduction while minimizing inband phase loss, with the optimal order and corner frequency dependent on the separation between the control bandwidth and the modes requiring gain reduction. The loop transfer function in Fig. 8 and the results in section 5b include a two-pole roll-off; if the structural damping is smaller so that greater separation is required, than a higher-order roll-off filter would be appropriate. From the loop transfer function shown for $p=2$, one would predict that a $2.5 \mathrm{~Hz}$ control bandwidth would be the maximum for a $6 \mathrm{~dB}$ gain margin. The actual stability limits will be lower due to the coupling between the dynamics associated with different Zernike basis vectors.

Because the global loop control objective is disturbance rejection, the relevant metric in comparing designs is the frequency at which the sensitivity transfer function is $-3 \mathrm{~dB}$ (a factor of $\sqrt{2}$ reduction in residual amplitude relative to uncontrolled), and this will be the definition of bandwidth used herein. With the additional phase lag from the filtering, this bandwidth can be significantly lower than the loop cross-over frequency.

\subsection{Multivariable Robustness Tools}

Stability can be predicted by computing eigenvalues of the closed-loop system, however, the eigenvalues do not provide any information about robustness. For a single-input, single-output system, typical robustness margins might be a $6 \mathrm{~dB}$ gain margin and $30^{\circ}$ phase margin. Satisfying these margins guarantees that at least at two particular frequencies, the loop transfer function is at least a distance of $1 / 2$ from the critical point of -1 , or equivalently that the magnitude of the sensitivity transfer function is less than 2 . In the multivariable case, requiring the peak magnitude $\left(H_{\infty}\right.$ norm) of the sensitivity to be less than 2 again gives a reasonable robustness margin in the absence of a specific understanding of the structure and magnitude of the uncertainty (see e.g. [12] for further details on the uncertainty that this guarantees robustness in the presence of). If the structure and segment dynamics transfer function is $G(j \omega)$ (from actuator force command to collocated position) with maximum singular value $\bar{\sigma}(j \omega)$ and the controller is $K(j \omega)$, then the robustness margin requirement is:

$$
\|S\|_{\infty}=\max _{\omega} \bar{\sigma}(S(j \omega)) \leq 2 \quad \text { where } \quad S=(1+G K)^{-1}
$$

\subsection{Servo loop interaction}

\section{TMT M1CS CSI}

The segment and servo dynamics are projected onto a Zernike basis, with basis functions up to radial degree 6 included (28 basis functions). Note that even though the M1 piston tip and tilt (Zernike radial degree 0 and 1 ) deflections are unobservable in the global control loop, there will be forces on the mirror aligned with these basis functions and therefore the servo loop will have non-zero response aligned with these basis functions, and must still be stable when coupled to the telescope structure; these basis functions are also included in the analysis.

For a particular PID actuator-servo control law, the eigenvalues of the coupled system with the actuator servo loops closed, but without the global loop closed, is shown in Fig. 9. The maximum singular value of the multivariable sensitivity is plotted in Fig. 11.

Structural damping of $0.5 \%$ is assumed, corresponding to the dominant line of closed-loop poles in the eigenvalue plot. Poles to the right of this $0.5 \%$ damping line have been destabilized by the control systems. The mount control system (MCS) also influences the M1 tip/tilt dynamics, but does not significantly influence the CSI stability boundary of the servo loop. The lowest frequency poles to the right of the $0.5 \%$ damping line, near $5 \mathrm{~Hz}$, are destabilized slightly by the MCS, and not by the actuator servo loops.

While the eigenvalue plot is sufficient to demonstrate stability, it is the maximum singular value of the sensitivity that is relevant to understanding the robustness of the control design to errors in the model. The robustness is limited by coupling with structural modes near $10 \mathrm{~Hz}$; this would not be evident from the eigenvalue plot alone. These modes are also low spatial-frequency structural, and project almost entirely onto the $p=2$ 


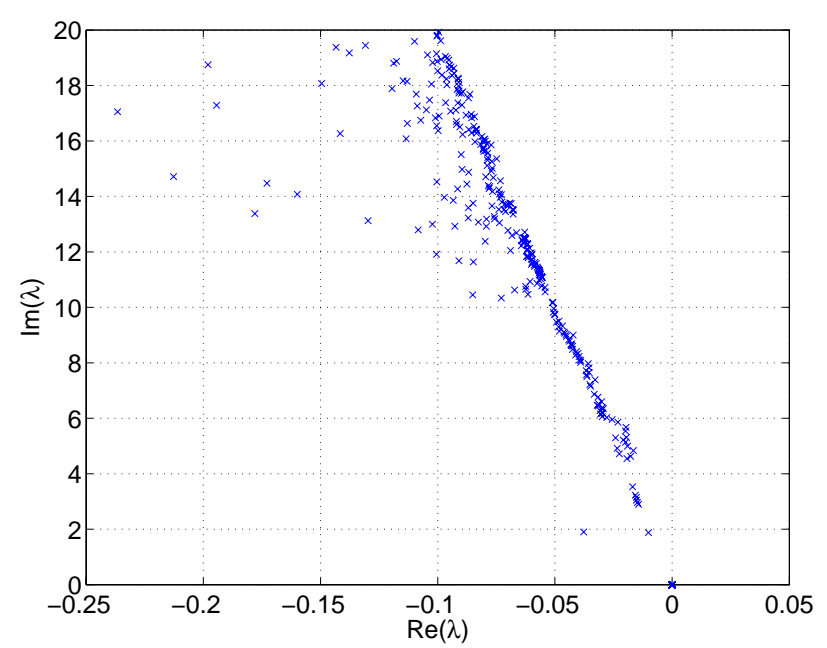

Figure 9. Stability of actuator servo loop: Closed-loop eigenvalues with servo loops closed but no global loops. Most of the shift in eigenvalues below $10 \mathrm{~Hz}$ is due to the mount control. The servo loop stabilizes modes $10-20 \mathrm{~Hz}$.

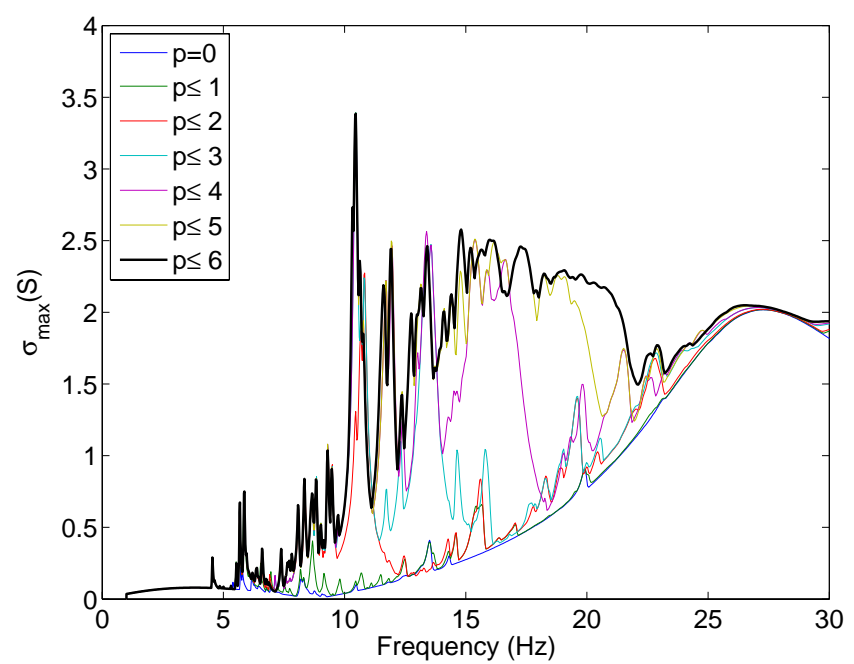

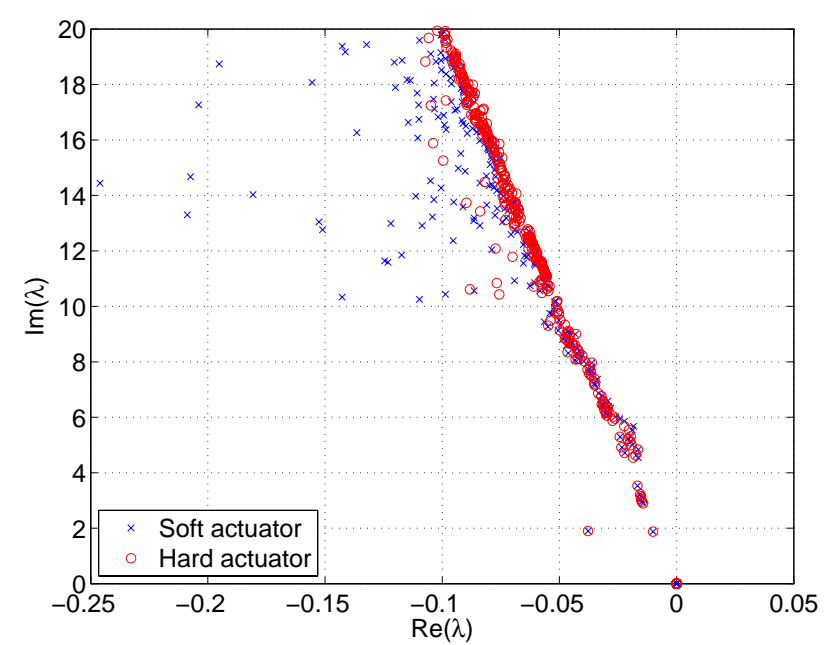

Figure 10. Stability of global control loop: Closed-loop eigenvalues, comparing hard and soft actuators with $1.5 \mathrm{~Hz}$ bandwidth. There is some influence of the global loop on poles above $10 \mathrm{~Hz}$, but the effect on poles below $10 \mathrm{~Hz}$ is not evident from the eigenvalues alone ( $c f$ Fig. 12).

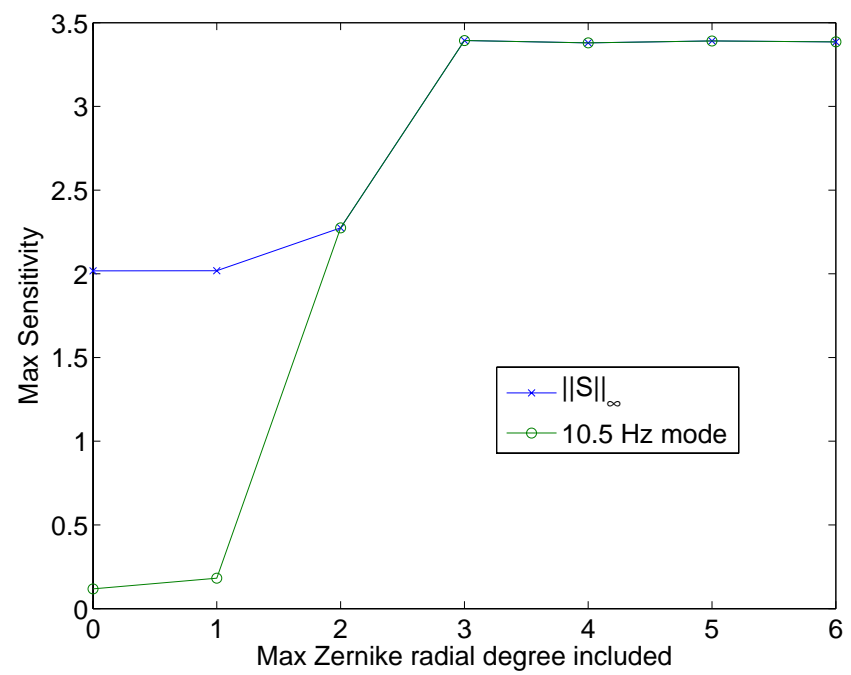

Figure 11. Robustness of actuator servo loop: maximum singular value of sensitivity for actuator servo loop (left), and dependency of maximum value on number of Zernike basis functions included (right). The servo design slightly exceeds $\|S\|_{\infty}=2$ without CSI, and exceeds significantly with CSI included. The robustness estimate converges with basis vectors up to radial degree 3 included.

and $p=3$ Zernike radial degrees, as evident from the right-hand plot in Fig. 11. There is no reason to expect that including the dynamics of the remaining $492-28$ basis functions would change the stability predictions.

The particular design analyzed here is clearly not sufficiently robust to model uncertainty, despite the assumed added damping, and further design iterations are required. Earlier servo controllers designed for a single segment mounted on a rigid base were unstable in the presence of CSI; with the $0.5 \%$ structural damping assumed here, stability required that the low frequency loop gain be reduced relative to that which gave the best performance on a rigid base. If the structural damping were increased to $1 \%$, then CSI issues would not limit the design of the servo loop. As noted earlier, because the servo loop is assumed to be collocated, stability could be guaranteed if a positive-real control design were used. However, this precludes the use of integral gain that is essential to obtaining sufficient low frequency stiffness. 

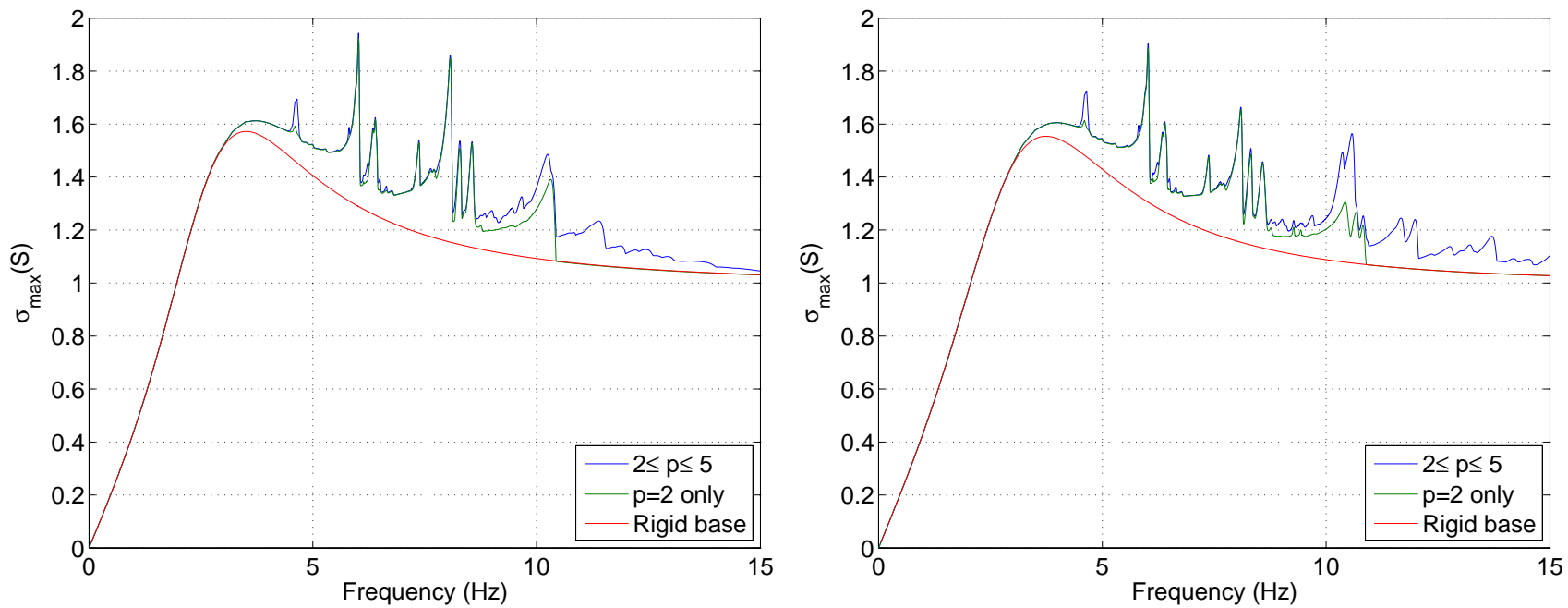

Figure 12. Maximum singular value of sensitivity for global control loop for soft (left) and hard actuator (right). Each plot compares the predicted robustness for only Zernike radial degree $p=2$, and for including all basis functions up to radial degree $p=5$; the maximum value is almost identical. The same controller is used on all modes, with a $-3 \mathrm{~dB}$ bandwidth of $1.5 \mathrm{~Hz}$.

\begin{tabular}{|c|c|c|c|c|} 
Radial & \multicolumn{3}{|c}{ Achievable Bandwidth $(\mathrm{Hz})$} & Limiting \\
degree & $\zeta=0.0025$ & $\zeta=0.005$ & $\zeta=0.01$ & Freq. (Hz) \\
\hline 2 & 1.3 & 1.55 & 1.75 & $6.0,8.1$ \\
3 & 1.9 & 1.9 & 1.95 & $4.6,5.8$ \\
4 & 1.85 & 1.9 & 1.95 & 4.6 \\
$5+$ & $>2$ & $>2$ & $>2$ & -
\end{tabular}

Table 1. Achievable control bandwidth (with $\|S\|_{\infty} \leq 2$ ) limited by CSI as a function of structural damping. A fixed 2-pole roll-off at $7 \mathrm{~Hz}$ is used which limits the bandwidth to $2.05 \mathrm{~Hz}$; for radial degree 5 and higher, the CSI constraint is higher than this frequency.

\subsection{Global loop interaction}

The CSI of the global loop performance is analyzed for both hard and soft actuators, again projecting onto Zernike basis of radial degree $p \leq 5$, and with global loop bandwidth of zero on the unobservable modes $p=0$ and $p=1$. For the soft actuators, analysis uses the servo loop analyzed in the previous section. The bandwidth achievable for the global control system is constrained by CSI. A higher bandwidth is possible for higher wavenumber degrees of freedom for which the structural coupling is small.

Figure 10 compares the eigenvalues of the coupled system using hard or soft actuation. As noted earlier, the eigenvalues alone demonstrate stability, but do not give any indication of robustness. The $H_{\infty}$ multivariable performance metric for the hard and soft actuators is plotted in Fig. 12. The characteristics are very similar. Using hard actuation, a slightly higher bandwidth is possible on the lowest radial degree modes, because the frequency shift due to the coupling is reduced (see Fig. 7), and a slightly lower bandwidth possible on higher radial degree modes because the soft actuator introduces some damping into the structural modes above roughly $10 \mathrm{~Hz}$. However, the quantitative behaviour is almost identical between the hard and soft actuators (the peak sensitivity in Fig. 10 is 1.90 and 1.92 respectively). This is not surprising, given the results in Fig. 8 illustrating the similar low frequency behaviour. Note that the highest peak in the sensitivity, near $6 \mathrm{~Hz}$ (a mainly astigmatic flexible mode of the mirror cell) is not evident in either the closed-loop eigenvalue plot nor the open-loop transfer plot. This illustrates that the Zernike basis is useful for reducing the number of degrees of freedom required in the analysis of stability and robustness, but it is not sufficient for representing the dynamics as a set of uncoupled single-input single-output problems.

The maximum bandwidth and relevant modal frequency for each of the lowest few Zernike radial degrees is shown in Table 1. To generate the table, the bandwidth (or gain) was separately modified for each Zernike radial degree in order to maintain $\|S\|_{\infty} \leq 2$. The table was generated for soft actuators, but the achievable bandwidth for hard actuators is not significantly different. The roll-off characteristics (2-pole at $7 \mathrm{~Hz}$ ) was kept constant for 
all of these, and this limits the bandwidth to $2 \mathrm{~Hz}$ independent of CSI; higher bandwidths would be possible for $p \geq 5$ with more careful design. (Note that a $2 \mathrm{~Hz}$ bandwidth corresponds to a $3.2 \mathrm{~Hz}$ loop crossover frequency.)

The predicted limit on achievable bandwidth due to CSI exceeds the required bandwidth. Note that sensor noise propagation will limit the bandwidth of the focus mode in particular to a value much lower than the limit due to dynamics.

\section{CONCLUSIONS}

The interaction between the TMT primary mirror control system and the telescope structural dynamics limits the achievable bandwidth of the M1CS global control. For 0.5\% structural damping, control-structure-interaction also limits the gain of the servo loop that is required to obtain sufficient low-frequency stiffness of a soft (voice-coil) type actuator. The servo gain would not be limited if the structure had $1 \%$ damping.

The key insight used to render the analysis computationally tractable is that the diagonal system of identical subsystems (segment dynamics and control) is diagonal under any change of basis, and thus the analysis can be conducted entirely in a Zernike basis. Because the support structure is more compliant on long length-scales than on short, the coupling and hence CSI are more significant for lower order Zernike shapes than for higher order. Using a modal control approach where the global control bandwidth is allowed to vary as a function of Zernike radial degree, then a $2 \mathrm{~Hz}$ bandwidth can be achieved on high wavenumber deformations of radial degree 5 and higher. Because low wavenumber deformations are more easily corrected by the adaptive optics system, as well as contributing less to seeing-limited performance, the use of modal control allows sufficient rejection of wind-induced response of M1 while maintaining adequate stability margins in the presence of control-structureinteraction (that is, the achievable bandwidth exceeds the required bandwidth).

The achievable bandwidth of the global control loop due to the dynamics is essentially the same for either a hard or a soft actuator. Note that other issues may result in lower feasible bandwidths than the CSI limit obtained here. In particular, sensor noise will limit the focus-mode bandwidth, and errors in the $A$ matrix may also require a significant reduction in the bandwidth of low spatial-frequency modes.

\section{Acknowledgements}

The TMT Project gratefully acknowledges the support of the TMT partner institutions. They are the Association of Canadian Universities for Research in Astronomy (ACURA), the California Institute of Technology and the University of California. This work was supported as well by the Gordon and Betty Moore Foundation, the Canada Foundation for Innovation, the Ontario Ministry of Research and Innovation, the National Research Council of Canada, the Natural Sciences and Engineering Research Council of Canada, the British Columbia

Knowledge Development Fund, the Association of Universities for Research in Astronomy (AURA) and the U.S. National Science Foundation.

\section{REFERENCES}

1. Aubrun, J.-N., Lorell, K. R., Havas, T. W., and Henninger, W. C., "Performance Analysis of the Segment Alignment Control System for the Ten-Meter Telescope," Automatica, Vol. 24, No. 4, pp. 437-453, 1988.

2. Jared, R. C., Arthur, A. A., Andreae, S., Biocca, A., Cohen, R. W., Fuertes, J. M., Franck, J., Gabor, G., Llacer, J., Mast, T., Meng, J., Merrick, T., Minor, R., Nelson, J., Orayani, M., Salz, P., Schaefer, B., and Witebsky, C., "The W. M. Keck Telescope segmented primary mirror active control system," Proc. SPIE Vol. 1236 Advanced Technology Optical Telescopes IV (Barr, L. D., ed.), 1990, pp. 996-1008.

3. Balas, M. J., "Trends in Large Space Structure Control Theory: Fondest Hopes, Wildest Dreams," IEEE Trans. on Automatic Control, Vol. 27, No. 3, pp. 522-535, 1982.

4. Aubrun, J.-N. and Lorell, K. R., "The Multi-Loop Control/Structure Interaction Effect: experimental verification using the ASCIE test bed," NASA/DoD CSI Conference, Nov 1990.

5. MacMynowski, D. G., Blaurock, C., and Angeli, G. Z., "Dynamic Analysis of TMT," Proc. SPIE, 2008. SPIE 7017-31.

6. Thompson, P. M., MacMynowski, D. G., and Sirota, M. J., "Control Analysis of the TMT Primary Segment Assembly," Proc. SPIE, 2008. SPIE 7012-58.

7. Chanan, G., MacMartin, D. G., Nelson, J., and Mast, T., "Control and Alignment of Segmented-Mirror Telescopes: Matrices, Modes, and Error Propagation," Applied Optics, Vol. 43, No. 6, pp. 1223-1232, 2004. 
8. Aubrun, J.-N., Lorell, K. R., Mast, T. S., and Nelson, J. E., "Dynamic Analysis of the Actively Controlled Segmented Mirror of the W. M. Keck Ten-Meter Telescope," IEEE Control Systems Magazine, pp. 3-9, Dec. 1987.

9. MacMynowski, D. G., Thompson, P. M., and Sirota, M. J., "Control of many coupled oscillators and application to segmented-mirror telescopes," AIAA Guidance, Navigation and Control Conference, 2008.

10. Szeto, K., Roberts, S., Gedig, M. H., Lagally, C., Tsang, D., MacMynowski, D. G., Sirota, M. J., Stepp, L. M., and Thompson, P. M., "TMT telescope structure system: design and development progress report," Proc. SPIE, 2008. SPIE 7012-88.

11. Thompson, P. M., MacMynowski, D. G., and Sirota, M. J., "Analysis of the TMT Mount Control System," Proc. SPIE, 2008. SPIE 7012-60.

12. Doyle, J. C., Francis, B. A., and Tannenbaum, A. R., Feedback Control Theory, MacMillan, 1992.

13. MacMartin, D. G. and Chanan, G., "Measurement accuracy in control of segmented-mirror telescopes," Applied Optics, Vol. 43, No. 3, pp. 608-615, 2004. 